\author{
FOLIA \\ Amazónica \\ Revista del Instituto de Investigaciones \\ de la Amazonía Peruana
}

\title{
EVALUACIÓN DE LAS TÉCNICAS DE APROVECHAMIENTO DE FRUTOS DE AGUAJE (Mauritia flexuosa L.f.) EN EL DISTRITO DE JENARO HERRERA, LORETO, PERÚ
}

\author{
LOURDES FALEN HORNA ${ }^{1,2}$, EURÍDICE N. HONORIO CORONADO² \\ 1 Universidad Nacional Agraria La Molina, Av. La Molina s.n., Lima, Perú. lourdesfalen@gmail.com \\ 2 Instituto de Investigaciones de la Amazonía Peruana, Av. Abelardo Quiñones km 2.5, lquitos, Perú.
}

\section{RESUMEN}

El aguaje es una palmera de alto valor comercial con importancia ecológica y social en la Amazonía Peruana. La tala excesiva de los individuos femeninos para el aprovechamiento de los frutos ha generado que la cosecha se realice cada vez a mayor distancia de los centros poblados y ha desencadenado la degradación y bajo estado de conservación de los aguajales en bosques naturales. El presente estudio tuvo como objetivo evaluar cómo los pobladores se vienen adaptando a la degradación del recurso, a través de la implementación de técnicas de aprovechamiento sostenible de frutos de aguaje. Aplicamos encuestas semi-estructuradas a 35 cosechadores de aguaje, evaluamos 17 áreas de aprovechamiento y acompañamos a 8 cosechadores en el aprovechamiento de los frutos de aguaje de 16 individuos adultos. Los resultados muestran que los pobladores del distrito de Jenaro Herrera han implementado técnicas de aprovechamiento que evitan la muerte de las plantas femeninas de menos de 20 metros de altura, es decir, hacen uso sostenible del recurso. Asimismo, el cultivo de la especie en los predios y huertas demuestra la preocupación de los pobladores por mantener este recurso más cerca de los centros poblados. Estas actividades desarrolladas demuestran la capacidad de adaptación de los pobladores locales ante la escasez de individuos. Por lo tanto, la voluntad de manejar el recurso genera nuevas oportunidades para la conservación y manejo sostenible del aguaje, asegurando así el abastecimiento de la demanda.

PALABRAS CLAVES: Aguajal, aprovechamiento sostenible, frutos amazónicos, producto forestal no maderable, subidor Maquisapa. 


\title{
ASSESSMENT OF THE TECHNIQUES USE TO HARVEST BURITI FRUITS (Mauritia flexuosa L.f.) IN THE DISTRICT OF JENARO HERRERA, LORETO, PERU
}

\begin{abstract}
The buriti is a palm of high commercial value, with ecological and social importancein the Peruvian Amazon. The excessive felling of the femaleindividuals for the use of the fruits has caused that the fruit harvest is carried out at greater distances from the population centres and has triggered the degradation and low state of conservation of the natural palm swamps. The objective of this study was to evaluate how the inhabitants are adapting to the degradation of the resource, through the implementation of techniques of sustainable use of buriti fruits. We applied semi-structured surveys to 35 buriti harvesters, evaluated 17 harvesting areas and accompanied 8 harvesters in the use of the buriti fruits of 16 adult individuals. The results show that the inhabitants of the district of Jenaro Herrera have implemented harvesting techniques that prevent the death of female plants less than 20 meters height, that is, they make sustainable use of the resource. In addition, the cultivation of the species in the farms and orchards demonstrates the concern of the inhabitants to keep this resource closer to the population centres. These activities demonstrate the ability of local people to adapt to the scarcity of buriti fruits. Therefore, the willingness to manage the resource generates new opportunities for the conservation and sustainable management of the buriti, thus ensuring the supply of demand.

KEYWORDS: Palm swamp, sustainable use, Amazonian fruits, non-timber forest product, Maquisapa climbing equipment.
\end{abstract}




\section{INTRODUCCIÓN}

La Amazonía peruana posee una alta riqueza de especies de palmeras, siendo algunas especies de gran importancia debido al elevado consumo de los frutos a nivel regional, como es el caso del aguaje, Mauritia flexuosa L.f. (Kahn, 1988). El aguaje es una palmera dioica, cuyas inflorescencias masculinas y femeninas se encuentran en plantas separadas, es decir, únicamente los aguajes femeninos son capaces de producir frutos (Manzi \& Coomes, 2009). En el 2012, se estima que la ciudad de Iquitos consumió casi 230,000 sacos de frutos de aguaje equivalentes a 8,206 toneladas métricas (Horn et al., 2018), y aproximadamente 57,500 individuos adultos aprovechados (4 sacos por individuo) (Freitas, 2012). El aprovechamiento de los frutos es casi siempre destructivo (Phillips, 1993) y se realiza a través de la tala cuando la planta mide más de 20 m (Holm et al., 2008); sin embargo, otros autores indican que la tala prevalece, aun cuando los frutos están lo suficientemente bajos como para usar una escalera (Vásquez \& Gentry, 1989). La alta demanda regional por los frutos de aguaje ha llevado a la reducción de la disponibilidad de los mismos en las cercanías a Iquitos (Delgado etal.,2007), dondegeneralmente se encuentran individuos masculinos y algunos individuos femeninos plantados. El decreciente acceso al recurso perjudica directamente a los cosechadores quienes deben caminar de tres a cuatro días para obtener los frutos (Vásquez \& Gentry, 1989). Por lo tanto, existe la necesidad de rescatar y promocionar técnicas locales de aprovechamiento sostenible de los frutos de aguaje para contribuir con la conservación de este recurso no maderable tan importante para la Amazonía peruana.

El aguaje crece generalmente en suelos inundados y puede alcanzar una alta densidad de individuos en ecosistemas denominados como aguajales (Vásquez \& Gentry, 1989). Los aguajales cubren más de 5 millones de hectáreas del territorio peruano y se distribuyen en los departamentos de San Martín, Amazonas, Huánuco, Madre de Dios, Cusco, Ucayali, Pasco y Loreto (Brako \& Zarucchi, 1993; Kahn et al., 1994). En Loreto, los aguajales ocurren en alta concentración a lo largo del río Marañón (Padoch, 1986) y cumplen un rol importante en la mitigación del cambio climático, además poseen una gran capacidad de almacenaje de carbono debajo del suelo (Householder et al., 2012; Draper et al., 2014; van Lent et al., 2018). El impacto de las prácticas destructivas se refleja en la estructura poblacional de los aguajales. Por ejemplo, en aguajales sobreexplotados la densidad de aguajes varía entre 50 y 160 individuos adultos por hectárea (Horn et al., 2012); de los cuales el 9\% de los adultos son individuos femeninos y la densidad de regeneración natural es baja. En aguajales no aprovechados, la densidad fluctúa entre 130 y 735 individuos adultos por hectárea (Kahn \& De Granville, 1992; Galeano et al., 2015; Honorio et al., 2015). La proporción estimada de individuos femeninos y masculinos en aguajales intervenidos o sobreexplotados en Perú es de 1:3.5 o 1:3.2 (Kahn, 1988; Horn et al., 2012), lo cual genera poblaciones inviables, es decir, no productivas (Vásquez \& Gentry, 1989); mientras que la proporción en aguajales intactos es de 1:1 (Kahn \& De Granville, 1992).

Cabe resaltar que existen experiencias de prácticas alternativas de cosecha sostenible en el departamento de Loreto. El método más simple utilizado es trepando directamente por el tallo usando los brazos y las piernas (Bernal \& Galeano, 2013). Cuando las palmeras presentan menos de $8 \mathrm{~m}$ de altura, los frutos son colectados desde el suelo, subiendo a través de escaleras, troncos auxiliares o apoyando el tallo de la caña Guadua angustifolia (Castaño-Arboleda etal.,2007; Bernal \& Galeano, 2013). Para la cosecha de individuos 
de más de $20 \mathrm{~m}$ de altura, se han desarrollado otras tecnologías. Por ejemplo, el estrobo es el sistema utilizado para escalar postes de luz, y está conformado por dos cuerdas que rodean el tallo de la palmera, cada extremo tiene una superficie para apoyar el pie y funciona balanceando el peso del cuerpo (Bernal \& Galeano, 2013). Esta técnica es usada en la comunidad de Parinari en el Río Marañón (Bejarano \& Piana, 2002; León et al., 2008), donde los hermanos Flores diseñaron su propio equipo, el cual se encuentra en un proceso continuo de mejora (León et al., 2008). Estos equipos fueron difundidos por la ONG CEDIA en la comunidad Roca Fuerte, entre el río Marañón y río San Luis, con una buena aceptación por parte de los pobladores, ya que les otorgó eficiencia en la cosecha, mejora de rendimientos y posibilitó regresar a las mismas áreas de aprovechamiento en la siguiente temporada (Manzi \& Coomes, 2009). Por su parte, las asociaciones ACORENA y COMAPA, pertenecientes a la comunidad Veinte de Enero en la Reserva Nacional Pacaya Samiria en la cuenca Yanayacu Pucate realizan el aprovechamiento sostenible del aguaje a través de la cosecha con equipos de escalada (Bernal \& Galeano, 2013). Otro ejemplo de cosecha sostenible es la realizada con el equipo de escalada Maquisapa, diseñado por la empresa Recursos Amazónicos Frutales S.A.C. Este equipo consta de dos cuerdas, un arnés y dos mosquetones y está complementado por una sierra telescópica. El subidor ha sido difundido en talleres organizados por el Instituto de Investigaciones de la Amazonía Peruana (IIAP) en el distrito Jenaro Herrera y por el Servicio Forestal de Fauna y Flora Silvestre en la región Loreto. Por otro lado, existen iniciativas de domesticación de la especie a través del cultivo en parcelas, como plantación o en sistemas agroforestales (Padoch, 1992) y particularmente en el cultivo de aguajes precoces para ofrecer frutos seleccionados a los mercados y disminuir las presiones en aguajes naturales (Del Castillo et al., 2010).

La pulpa de los frutos de aguaje es muy apreciada en la Amazonía peruana por su agradable sabor y se aprovechan para el consumo directo, o para su comercialización a empresas que elaboran productos secundarios como pasta seca, bebidas y helados (Delgado et al., 2007). Asimismo, la composición química revela un alto contenido de ácidos grasos, proteína y fibras; lo que ha despertado el interés de las industrias alimenticias, farmacéuticas y cosméticas en los mercados internacionales por ser un alimento energético, fuente de beta-caroteno y vitamina E (Pacheco Santos, 2005; Darnet et al., 2011). El aguaje no solo es una fuente de alimento para los pobladores, también les provee fibras, medicina y material para construcción y confección de artesanías (Balslev et al., 2008). Las bondades de esta especie no culminan cuando la planta ha muerto, pues en los troncos caídos incuban larvas de Rhynchophorus palmarum (Coleoptera: Curculionidae), una importante fuentealimenticia para el poblador rural en la región amazónica (Hiraoka, 1999; Balslev et al., 2008). Por lo tanto, el presente estudio tuvo como objetivo evaluar cómo los pobladores del distrito de Jenaro Herrera se vienen adaptando a la degradación del recurso, a través de la implementación de técnicas de aprovechamiento sostenible de frutos de aguaje. Los objetivos específicos del estudio fueron (1) conocer las características del aprovechamiento y las técnicas de cosecha de frutos de aguaje en el distrito de Jenaro Herrera; (2) evaluar el estado de conservación de las áreas de aprovechamiento del recurso y (3) los tiempos utilizados en las diferentes etapas del aprovechamiento de los frutos. 


\section{MATERIAL Y MÉTODOS}

\section{ÁREA DE ESTUDIO}

El estudio se realizó en el distrito de Jenaro Herrera (04⒌'19"S $\left.73^{\circ} 40^{\prime} 22^{\prime \prime} 0\right)$, provincia de Requena, departamento de Loreto, e incluyó Villa Jenaro Herrera y los centros poblados de Nuevo Progreso, Nuevo San Juan, Nuevo Aucayacu y Once de Agosto. La temperatura media anual de la zona es de $26.9^{\circ} \mathrm{C}$ y la precipitación media anual de 2,730 mm, la época de vaciante de los ríos ocurre entre junio y octubre y coincide con la temporada menos lluviosa (Ríos et al., 1974). La población del distrito es de alrededor de 5,561 habitantes, cuyas principales actividades económicas son la agricultura, pesca, caza y extracción de recursos naturales (Kvist \& Nebel, 2001).

Los bosques de altura de Jenaro Herrera ocurren en la planicie interfluvial con presencia de pequeños parches de bosques de arena blanca; mientras en la planicie fluvial del río Ucayali, existen grandes bosques estacionalmente inundados y aguajales (Kvist \& Nebel, 2001). Los aguajales son extensos en las zonas inundables; no obstante, también ocurren en pequeños a medianos parches dentro de los bosques de altura, particularmente en zonas de mal drenaje (López Parodi \& Freitas, 1990). En la actualidad, los aguajales aledaños a Villa Jenaro Herrera han sido depredados, quedando pocos relictos naturales productivos y accesibles a través de la carretera Jenaro Herrera - Angamos. En nuestro estudio, realizamos las evaluaciones tanto en aguajales de origen natural y también en aquellos aguajes plantados en parcelas y huertas en Villa Jenaro Herrera y en los centros poblados visitados.

\section{LA PALMERA DE AGUAJE}

El aguaje, Mauritia flexuosa L.f., es conocido como burití, moriche, morete y palmera real en Brasil, Venezuela, Ecuador y Bolivia, respectivamente
(Delgado et al., 2007; Manzi \& Coomes, 2009). Se distribuye ampliamente en la cuenca Amazónica y Centroamérica, cubriendo Perú, Bolivia, Colombia, Ecuador, Venezuela, Brasil, Las Guyanas, Trinidad y Panamá (Henderson et al., 1995). Es una palmera dioica, tiene fuste recto $y$ cilíndrico y puede alcanzar $35 \mathrm{~m}$ de altura y $50 \mathrm{~cm}$ de diámetro. Posee hojas costapalmadas de más de $5 \mathrm{~m}$ y un peciolo de 1.4 a $4 \mathrm{~m}$ de longitud. No se han evidenciado características morfológicas que permitan distinguir plantas femeninas de las masculinas antes de la primera floración, que ocurre aproximadamente a los 7 años (Villachica et al., 1996; Delgado et al., 2007). Las plantas femeninas producen entre 4 y 8 racimos de 2 a $3 \mathrm{~m}$ de largo, cada uno con alrededor de 900 frutos (Delgado et al., 2007). El fruto es una drupa elipsoidal, el color varía entre anaranjado oscuro y marrón rojizo y mide de 5 a $7 \mathrm{~cm}$ de longitud (Henderson et al., 1995). La semilla es recalcitrante y presenta dormancia pronunciada (Silva et al., 2014). El aguaje es una especie anemófila (Khorsand Rosa \& Koptur, 2013) y es dispersada por tapires, venados, primates, roedores, aves y peces (Bodmer, 1991; Macedo, 2002; Lees \& Peres, 2008; Virapongse et al., 2017).

Generalmente la etapa reproductiva del aguaje es estacional y varía en las diferentes cuencas de la Amazonía (Horn et al., 2018). El pico más alto de la floración se da cuando los niveles de inundación empiezan a disminuir (Khorsand Rosa et al., 2013); por el contrario, el pico de la fructificación ocurre en la estación húmeda (Díaz et al., 2015; Virapongse et al., 2017). En el distrito de Jenaro Herrera, el aprovechamiento de los frutos de aguaje ocurre entre los meses de mayo a julio.

\section{COLECTA DE DATOS}

El trabajo de campo fue realizado entre los meses de mayo a agosto del 2018. Para conocer las características del aprovechamiento de frutos de 

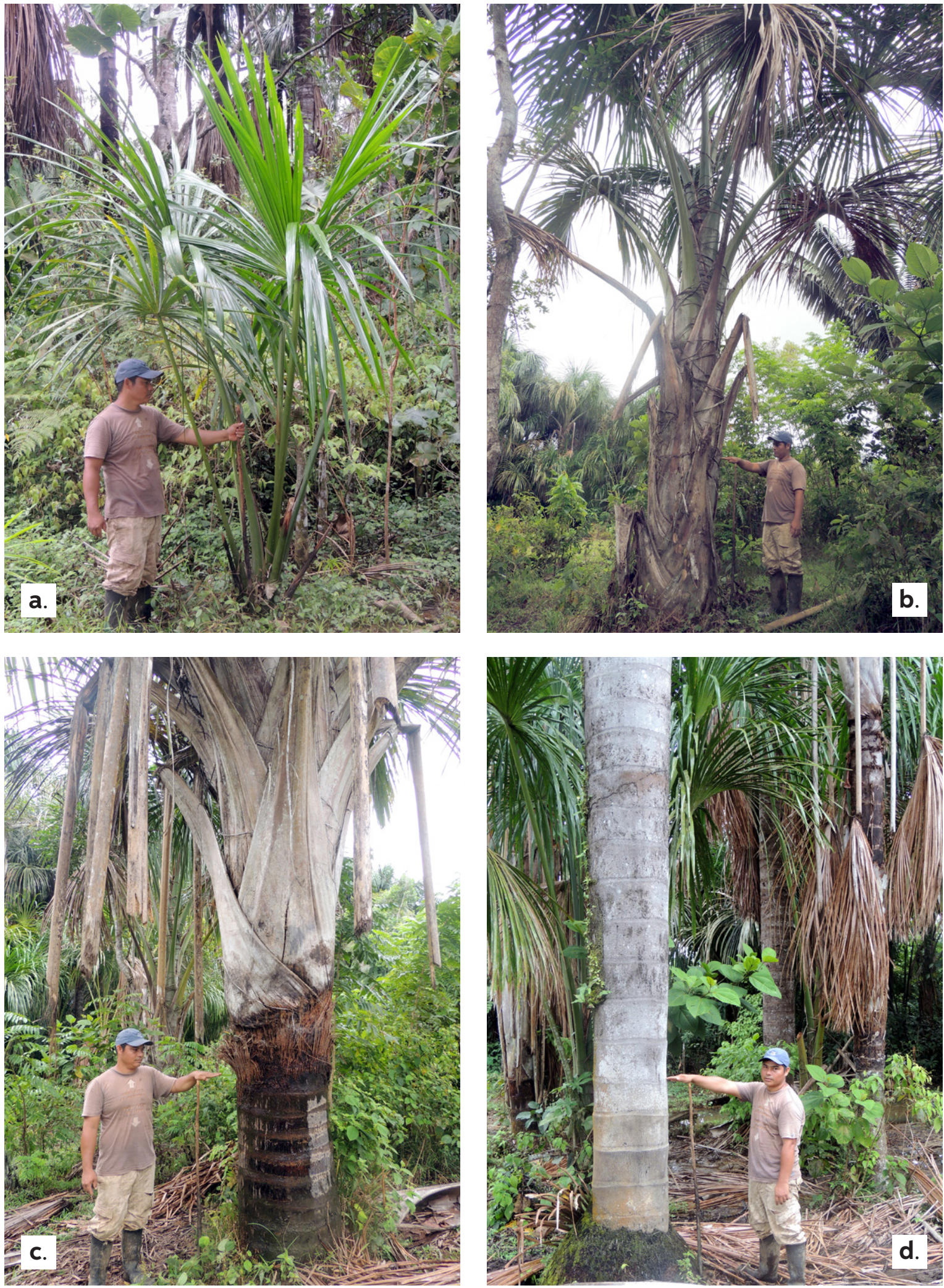

Figura 1. Categorías de crecimiento del aguaje utilizadas en las evaluaciones de los predios y relictos de aguaje manejados en el distrito de Jenaro Herrera. (a-c) Estadío juvenil y (d) Estadío adulto. 
aguaje aplicamos encuestas semiestructuradas a 35 cosechadores de aguaje, a quienes definimos como personas que aprovechan los frutos durante la temporada de fructificación con el propósito de comercializar el producto o para consumo propio. La encuesta buscó obtener información socioeconómica como género, edad, residencia, número de miembros del hogar $\mathrm{y}$ actividades productivas. Se registró la ubicación espacial de las áreas de aprovechamiento, la temporada de cosecha de frutos, las técnicas de cosecha, las razones de preferencia de algunas técnicas, la producción anual y vías de comercialización de los frutos de aguaje. También recogimos información de la percepción de los cosechadores sobre la disponibilidad del recurso.

Seleccionamos a 17 cosechadores para evaluar las características de las áreas de aprovechamiento del recurso. Consideramos como áreas de aprovechamiento las zonas que los encuestados reportaron como lugares donde ejercen dicha actividad, ya sean aguajales naturales o aguajes plantados en predios. La presencia de individuos juveniles y femeninos fue utilizada como indicador del estado de conservación en las áreas de aprovechamiento por lo que censamos y georreferenciamos los individuos de aguaje juveniles y adultos femeninos y masculinos. Clasificamos a los individuos por estadíos (Figura 1), consideramos individuos juveniles a aquellos que presentaron al menos 2 m de altura sin presencia de tallo (a), con tallo cubierto por peciolos de hojas o tallo visible de menos de $1.30 \mathrm{~m}$ (b) y con tallo visible de más de $1.30 \mathrm{~m}$, pero sin evidencias fenológicas de su sexo (c); mientras que los adultos fueron aquellos individuos con tallo visible de más de $1.30 \mathrm{~m} \mathrm{y}$ con evidencias fenológicas de su sexo (d).

Asimismo, 8 cosechadores encuestados ofrecieron demostrar las técnicas empleadas en el aprovechamiento del aguaje, por lo que observamos la cosecha de 16 individuos adultos de aguaje. Categorizamos las técnicas, medimos los tiempos de duración de las etapas de aprovechamiento del aguaje (ascenso, corte, descenso y desgranado-embolsado) y contamos el número de participantes de acuerdo a los roles que desempeñan en el proceso. Incluimos mediciones de la altura total de la palmera, altura de fuste, número total de racimos, número de racimos aprovechados por palmera, peso de 20 frutos por racimo que pesamos individualmente en una balanza digital de precisión $0.1 \mathrm{~g}$, peso total de los frutos desgranados por cada racimo pesados con una balanza romana de precisión de $0.5 \mathrm{~kg}$, y número de sacos obtenidos por individuo de aguaje cosechado.

\section{PROCESAMIENTO DE LA INFORMACIÓN}

Las encuestas y las evaluaciones fueron digitalizadas en una hoja de cálculo de Microsoft Excel y fueron procesadas en el mismo programa para obtener valores promedios y la desviación estándar de los datos (cuando fue posible calcularlos). La información de tipo cualitativa fue representada en gráficos de barras.

\section{RESULTADOS}

\section{CARACTERÍSTICAS DEL APROVECHAMIENTO DE LOS FRUTOS DE AGUAJE}

De los 35 cosechadores de aguaje entrevistados, 14 fueron mujeres y 21 varones con edades entre los 30 y 67 años, pertenecientes a Villa Jenaro Herrera (63\%), y comunidades Nuevo San Juan (14\%), Nuevo Aucayacu (11\%), Nuevo Progreso (9\%) y Once de Agosto (3\%). Los hogares de los encuestados están conformados por un promedio de 4 miembros (rango: 1 - 8 miembros). La principal actividad económica de los cosechadores de aguaje es la agricultura (80\%), seguida de la cacería y la pesca (11\%) y otras actividades (9\%). 
Los cosechadores mencionaron 42 áreas de aprovechamiento en el distrito de Jenaro Herrera, las cuales fueron visitadas de 1 a 8 veces al año. Estas áreas de aprovechamiento suelen estar cercanas a los centros poblados de la procedencia de los cosechadores (Figura 2). A través de la verificación en campo, detectamos que alrededor del $50 \%$ de las áreas están en formaciones naturales y otro $50 \%$ en predios donde cuentan con uno a 40 individuos de aguaje generalmente plantados. Las áreas de aprovechamiento en formaciones naturales reportadas tienen diferentes características y niveles de conservación (Figura 3); por ejemplo, tenemos un aguajal natural cercano a la quebrada Fierrillo donde indican los pobladores locales fue una zona de extracción intensiva de aguaje a finales de los setentas y en la actualidad solo se encuentran individuos adultos masculinos en pie y regeneración natural casi nula (a). Junto a la carretera Jenaro Herrera - Angamos, existen áreas de aprovechamiento en relictos de aguajales dentro de pastizales, donde se han mantenido algunos individuos productivos de aguaje y hay una alta proporción de plantas masculinas y alguna regeneración natural (b). Asimismo, encontramos un fragmento de aguajal bajo manejo dentro de Villa Jenaro Herrera, en donde la proporción de individuos femeninos y masculinos es casi equivalente y existe gran cantidad de regeneración natural (c).

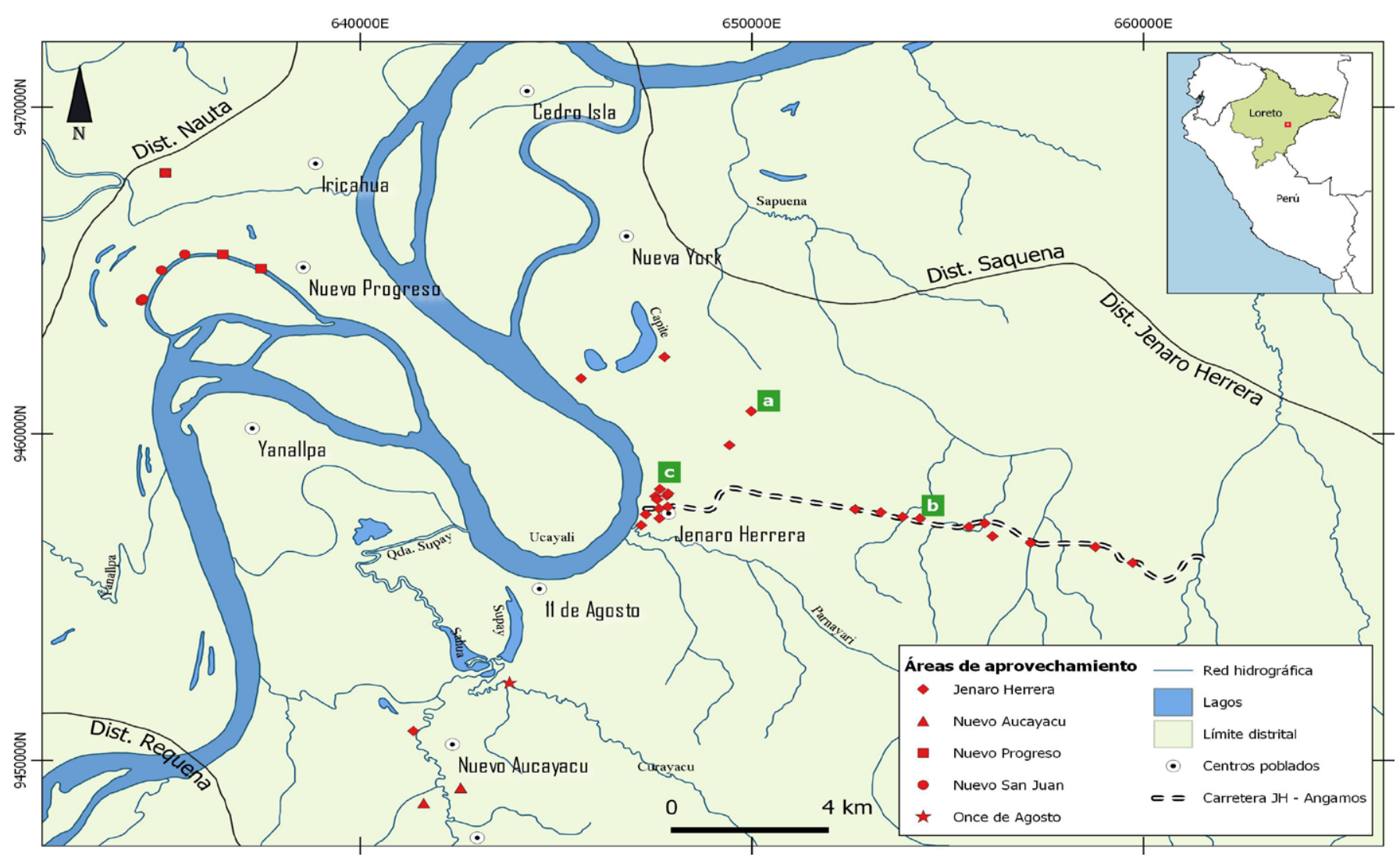

Figura 2: Ubicación de las 42 áreas de aprovechamiento de aguaje reportadas en el distrito de Jenaro Herrera. El mapa interno muestra la ubicación de la zona de estudio dentro del departamento de Loreto (Datum WGS84, Proyección UTM Zona 18L Sur). Las letras a-c indican la ubicación de las áreas de aprovechamiento mostradas en Figura 3. 

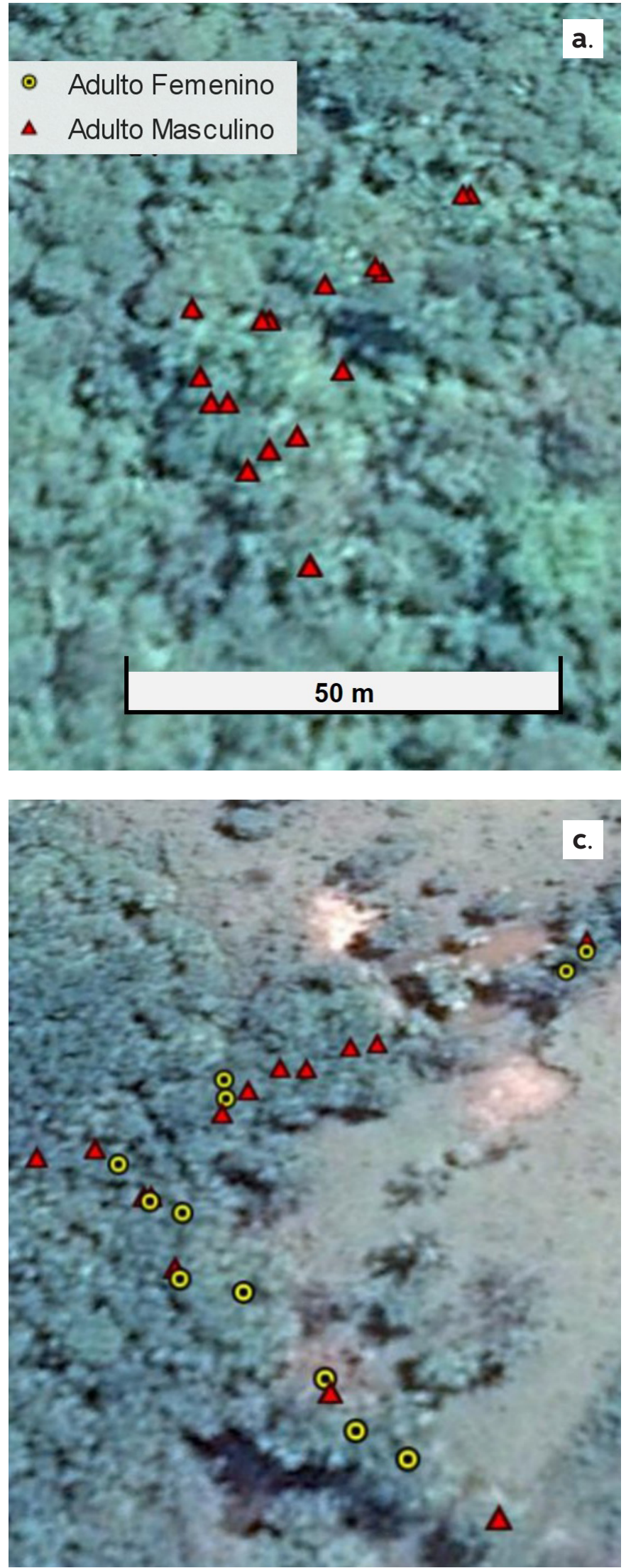

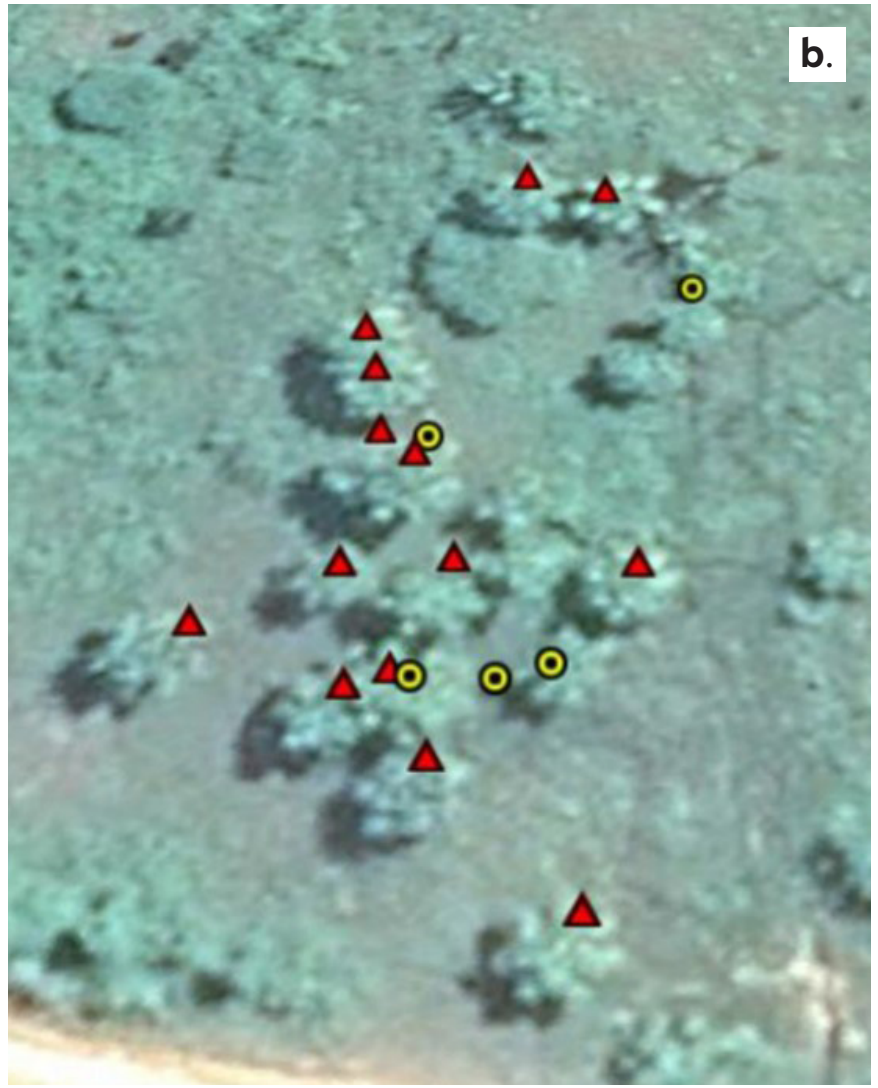

Figura 3: Vista aérea de tres áreas de aprovechamiento visitadas durante el trabajo de campo en Villa Jenaro Herrera, indicando la ubicación de los individuos adultos masculinos y femeninos. (a) Aguajal quebrada Fierrillo, (b) aguajal remanente dentro del pastizal del Sr. José Pisango en el km 7.3 de la carretera Jenaro Herrera - Angamos, (c) aguajal remanente manejado por el Sr. Ramiro Valles en el km 0.1 de la misma carretera. Imágenes obtenidas de Google Earth. 

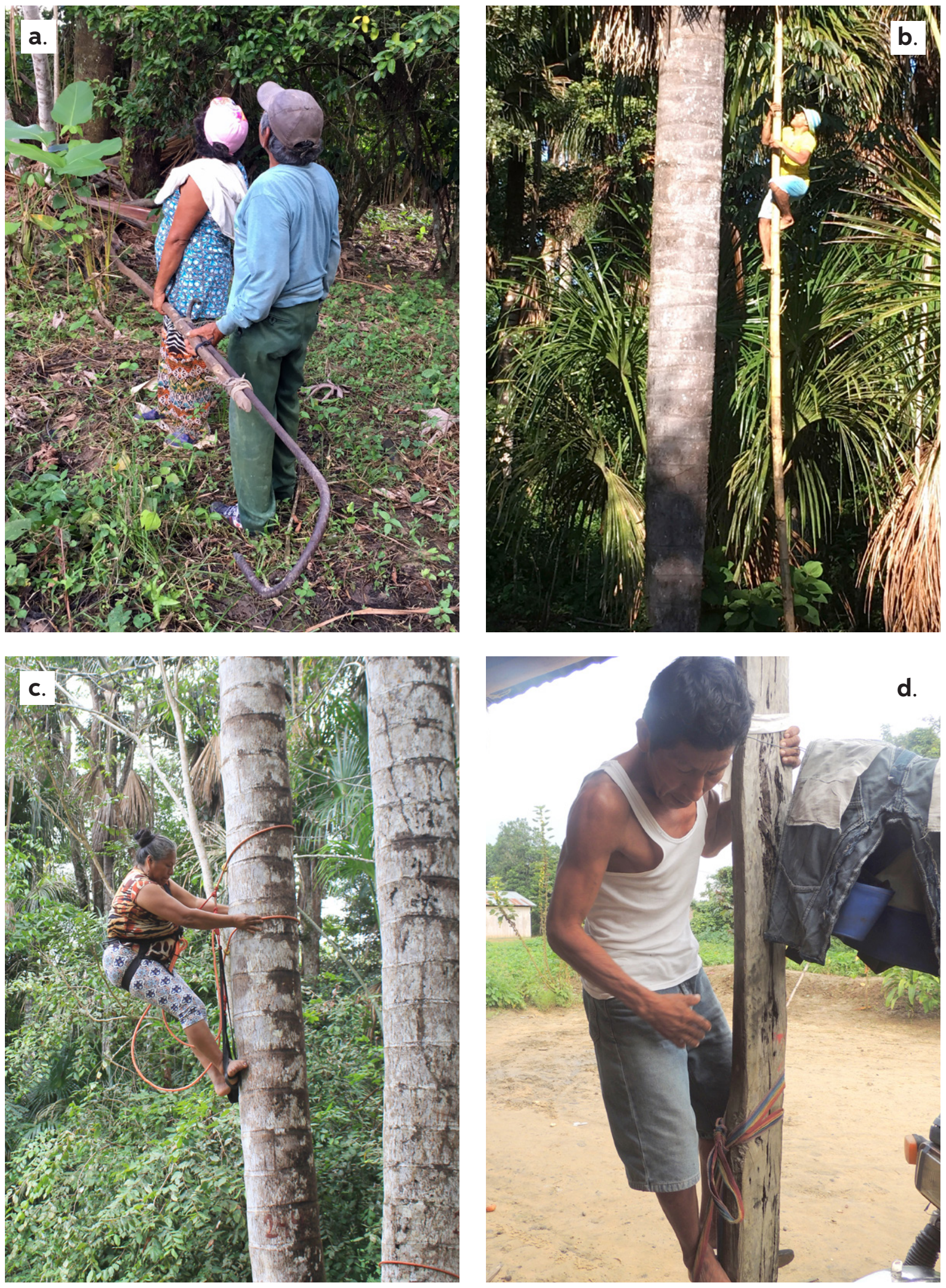

Figura 4: Técnicas de cosecha de aguaje utilizadas por los pobladores de Villa Jenaro Herrera. (a) Vara de madera con accesorio de metal, (b) vara de caña con accesorio de madera, (c) equipo de escalada Maquisapa, (d) pretina. 
Los cosechadores entrevistados manejan de 1 a 2 tipos de técnicas de cosecha. Las técnicas de cosecha (Figura 4) pueden ser agrupadas en cinco tipos:

\section{La vara con o sin accesorios}

Es la técnica más reportada y consiste en una vara que se apoya en el tronco de la palmera y por el cual el cosechador asciende hasta alcanzar los racimos. La vara puede ser de madera y tener una horquilla o complementarse con un gancho de metal o madera en el extremo superior (a) que se engancha en el peciolo de la hoja de la palmera para dar estabilidad a la vara. Además, usan sogas o pretinas para asegurar la vara. Otro tipo de vara es la caña de Guadua angustifolia (b), que cuenta con espinas largas en cada entrenudo, y que son usadas a modo de peldaño durante el ascenso. El elemento de corte más usado para el aprovechamiento de los racimos es el machete.

\section{Equipo de escalada o subidor Maquisapa}

Está conformado por un arnés de seguridad, dos mosquetones y dos cuerdas de materiales especializados (c). El arnés se coloca regulando el cinturón a las medidas del cosechador, las cuerdas se entrelazan en el fuste de la palmera y se enganchan al mosquetón con rosca, la línea de vida se coloca en el otro mosquetón. El sistema de ascenso se basa en la distribución del peso en cada pie y los movimientos son similares a subir una escalera. El arnés presenta un cinturón en el cual se puede enganchar una sierra telescópica. Incluimos en esta categoría un subidor artesanal elaborado con soga de monte que presenta características similares al Maquisapa.

\section{La pretina}

Es la técnica que emplea una o más pretinas, se amarran sus extremos y se coloca en los tobillos, estirando los pies y apoyando la pretina en el tronco, simultáneamente se debe ascender usando los brazos (d). El elemento de corte generalmente es el machete.

\section{Trepado sin equipo}

Es la técnica más simple, que consiste en ascender el tallo del aguaje usando la fuerza de los brazos y piernas de manera coordinada sin ningún equipo adicional. Observamos esta técnica una sola vez y fue ejecutada por el Sr. Ramiro Valles de Villa Jenaro Herrera.

\section{La tala}

Es la técnica más común en el aprovechamiento de frutos de aguaje en Amazonía, en particular cuando los frutos se encuentran a alturas considerables. Se realiza con un hacha y es una de las causas de la disminución de aguajales productivos en bosques naturales.

Los cosechadores que usan las técnicas de trepado sin equipo, la vara con o sin accesorios, la pretina y el equipo de escalada prefieren estos métodos porque les permiten la conservación de sus recursos; en el caso del equipo de escalada mencionaron que también les proporciona seguridad durante el ascenso. Si bien los 35 cosechadores encuestados usaron alguna de estas técnicas de cosecha sostenible, ocho de ellos también aprovecharon los frutos de aguaje cortando el tallo con un hacha. En el caso de 13 aguajeros encuestados, reportaron realizar el aprovechamiento contratando a terceros para realizar la cosecha de los frutos a través de técnicas sostenibles. Sin embargo, los cosechadores que deciden talar las plantas de aguaje indican que lo hacen por conveniencia, es decir cuando es la alternativa más rápida y fácil en ese momento, generalmente porque los frutos están muy altos.

La temporada de cosecha de frutos inicia generalmente en mayo y culmina entre julio y agosto, es decir tiene una duración de tres a cuatro meses; sin embargo, algunos 


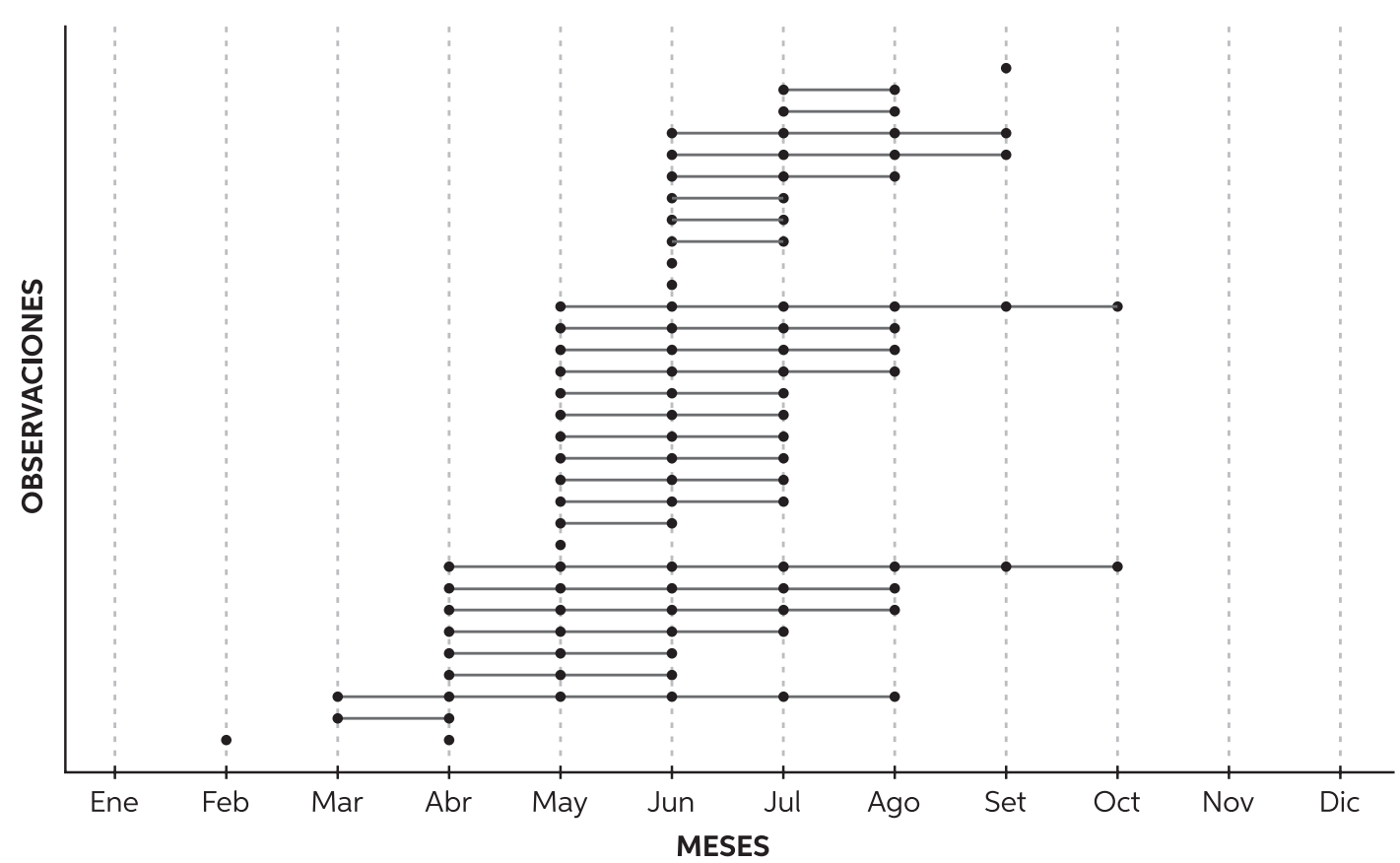

Figura 5: Temporada de aprovechamiento de aguaje reportada por 32 cosechadores encuestados en el distrito de Jenaro Herrera. Tres cosechadores declararon desconocer la temporada de cosecha.

cosechadores indican periodos más cortos y otros periodos más extensos (Figura 5). Durante la cosecha del año 2017, estimamos que al menos 28 cosechadores encuestados aprovecharon 307 palmeras (promedio: 10 palmeras; rango: 1-150 palmeras) que llenaron 1,110 sacos de aguaje (promedio: 38 sacos; rango: 1-600 sacos). El principal objetivo de la cosecha de aguaje fue la venta de los frutos; sin embargo, también reservaron una parte para el consumo propio. El precio de venta mínimo de un saco fue de S/. 15, mientras que en la época de escasez de frutos el precio se elevó hasta S/. 70 por saco. Por otro lado, 13 cosechadores indicaron que venden frutos de aguaje al menudeo, 5 a 6 frutos pelados por S/. 1; esta actividad es realizada principalmente por las mujeres encuestadas y por las esposas de los cosechadores.

Al menos el $60 \%$ de los cosechadores encuestados coinciden en notar una menor disponibilidad de frutos de aguaje respecto al pasado; asimismo, afirman que es necesario recorrer distancias mayores para encontrar individuos productivos.

\section{ÁREAS DE APROVECHAMIENTO DEL AGUAJE}

Casi todas las áreas de aprovechamiento evaluadas fueron predios constituidos por huertas o chacras en las que los individuos de aguaje son de origen natural y/o plantado, generalmente acompañados por otros cultivos y están ubicados en Villa Jenaro Herrera, Nuevo Progreso y Nuevo San Juan (Figura 6). En estos 16 predios evaluamos 68 individuos juveniles y 86 adultos ( 45 femeninos y 41 masculinos), y los cosechadores mostraron tener de 1 a 7 individuos femeninos aprovechables que producen en promedio 4.6 racimos por planta (rango: 1-10 racimos).

El predio manejado por el Sr. Ramiro Valles, está conformado por un fragmento de aguajal que fue mantenido con fines de aprovechamiento. Dicho predio cuenta con presencia de alta regeneración natural por lo que se hizo un muestreo usando 

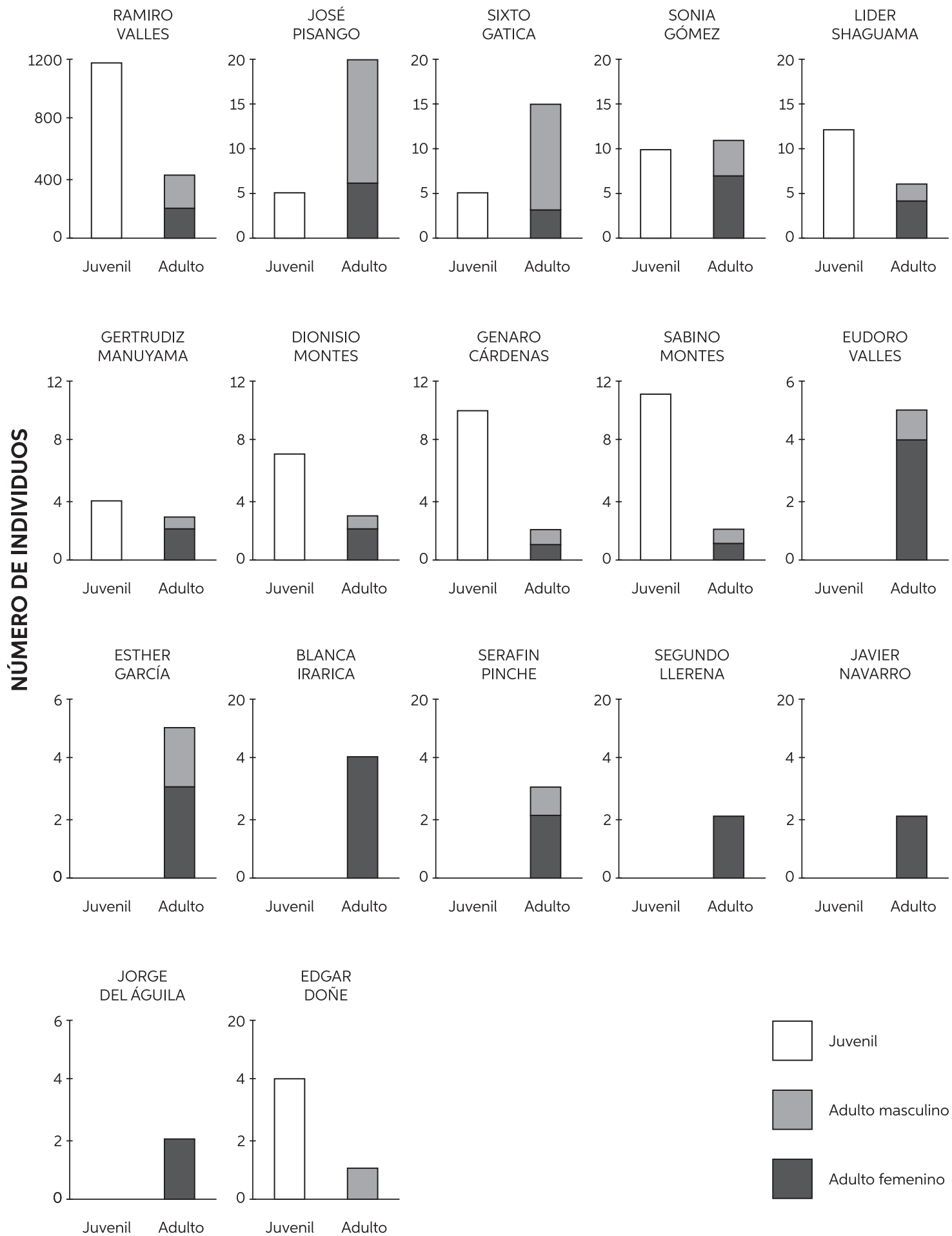

Figura 6: Número de individuos de aguaje según categorías de crecimiento evaluados en 17 áreas de aprovechamiento en el distrito de Jenaro Herrera. Los señores Ramiro Valles, Sonia Gómez y José Pisango manejan tres, una y dos hectáreas de aguajales, respectivamente; mientras que el resto de personas evaluadas cosechan el aguaje dentro de sus predios en áreas menores a 0.5 hectáreas. 
Tabla 1: Duración de cada etapa del aprovechamiento de los frutos de 16 individuos de aguaje según las diferentes técnicas de cosecha observadas en Villa Jenaro Herrera.

\begin{tabular}{|l|c|c|c|c|}
\hline \multirow{2}{*}{ Técnica de cosecha } & \multicolumn{4}{|c|}{ Tiempo en minutos } \\
\cline { 2 - 5 } & Ascenso & Corte & Descenso & Desgr.-empaq. \\
\hline Equipo de escalada $(n=12)$ & $\begin{array}{c}13.3 \pm 7.6 \\
(6.0-28.0)\end{array}$ & $\begin{array}{c}14.1 \pm 10.9 \\
(1.0-37.0)\end{array}$ & $\begin{array}{c}7.0 \pm 5.2 \\
(2.0-20.0)\end{array}$ & $\begin{array}{c}64.8 \pm 40.6 \\
(13.0-143.0)\end{array}$ \\
\hline Vara con o sin accesorios $(n=3)$ & $\begin{array}{c}5.3 \pm 4.3 \\
(1.0-9.5)\end{array}$ & $\begin{array}{c}6.0 \pm 7.8 \\
(1.5-15.0)\end{array}$ & $\begin{array}{c}5.7 \pm 5.0 \\
(1.0-11.0)\end{array}$ & $\begin{array}{c}40.3 \pm 4.5 \\
(36.0-45.0)\end{array}$ \\
\hline Trepado sin equipo $(n=1)$ & $1.3 \pm N A$ & $4 \pm N A$ & $2.5 \pm N A$ & $49 \pm N A$ \\
\hline
\end{tabular}

NA, no aplica

Tabla 2: Características y productividad de frutos de los 16 individuos de aguaje cosechados por pobladores locales en Villa Jenaro Herrera.

\begin{tabular}{|c|c|c|c|c|c|c|c|}
\hline $\mathbf{N}^{\circ}$ & $\begin{array}{c}\text { Altura de } \\
\text { fuste }(\mathbf{m})\end{array}$ & $\begin{array}{c}\text { Altura } \\
\text { total }(\mathbf{m})\end{array}$ & $\begin{array}{c}\text { Racimos } \\
\text { totales }\end{array}$ & $\begin{array}{c}\text { Racimos } \\
\text { cosechados }\end{array}$ & $\begin{array}{c}\text { Peso del } \\
\text { racimo (kg) }\end{array}$ & $\begin{array}{c}\text { Peso del fruto } \\
(\mathbf{g})^{*}\end{array}$ & $\begin{array}{c}\mathbf{N}^{\circ} \text { de } \\
\text { sacos }\end{array}$ \\
\hline 1 & 13.5 & 18 & 7 & 5 & $33.0 \pm 9.9$ & $63.2 \pm 4.2$ & 3.3 \\
\hline 2 & 12 & 17 & 7 & 1 & $18.0 \pm N A$ & $52.1 \pm \mathrm{NA}$ & 0.3 \\
\hline 3 & 13 & 18 & 4 & 4 & $38.6 \pm 9.9$ & $46.7 \pm 1.4$ & 3.5 \\
\hline 4 & 11 & 15 & 4 & 4 & $26.7 \pm 10.8$ & $39.8 \pm 2.9$ & 2.3 \\
\hline 5 & 6.5 & 13 & 5 & 5 & $22.4 \pm 9.7$ & $57.6 \pm 5.4$ & 2 \\
\hline 6 & 15 & 20 & 4 & 3 & $20.0 \pm 1.6$ & $47.8 \pm 4.2$ & 1.3 \\
\hline 7 & 11 & 14 & 7 & 4 & $40.0 \pm 7.7$ & $45.7 \pm 4.0$ & 4 \\
\hline 8 & 9.5 & 13 & 4 & 4 & $9.2 \pm 9.2$ & $52.6 \pm 2.0$ & 1 \\
\hline 9 & 10 & 15 & 3 & 3 & $43.6 \pm 9.2$ & $61.6 \pm 2.3$ & 3 \\
\hline 10 & 14 & 17 & 4 & 4 & $23.2 \pm 1.1$ & $44.9 \pm 2.4$ & 2.3 \\
\hline 11 & 3 & 11 & 5 & 5 & $21.1 \pm 2.4$ & $55.9 \pm 6.9$ & 2.7 \\
\hline 12 & 13 & 17 & 8 & 6 & $29.2 \pm 5.3$ & $44.5 \pm 0.7$ & 4.5 \\
\hline 13 & 11 & 18 & 4 & 2 & $19.0 \pm N A$ & $39.8 \pm N A$ & 1 \\
\hline 14 & 10.5 & 18 & 5 & 1 & $33.5 \pm N A$ & $38.2 \pm N A$ & 0.5 \\
\hline 15 & 13 & 20 & 5 & 5 & $22.0 \pm 15.8$ & $57.1 \pm 12.3$ & 1.5 \\
\hline 16 & 10 & 18 & 6 & 2 & $18.2 \pm N A$ & $37.4 \pm N A$ & 1 \\
\hline
\end{tabular}

NA, no aplica; *, valores de promedio y desviación estándar fueron obtenidos del peso de 20 unidades de frutos por racimo. 
dos parcelas de $10 \mathrm{~m}$ x $100 \mathrm{~m}$, para después estimar el total de individuos para las tres hectáreas manejadas. En total, encontramos 1,170 individuos juveniles y 420 individuos adultos (195 femeninos y 225 masculinos) con un promedio de 3.1 racimos por planta (rango: 1-5 racimos).

\section{ETAPAS EN EL APROVECHAMIENTO DEL AGUAJE}

Durante la evaluación de la cosecha de 16 individuos productivos de aguaje identificamos que esta actividad presenta cuatro etapas que incluyen el ascenso a la palmera, el corte de los racimos, el descenso y el desgranadoempaquetado de los frutos. Logramos observar la cosecha de 12 individuos con equipo de escalada, tres individuos cosechados con vara con o sin accesorios y un individuo cosechado trepando sin equipo. El uso del equipo de escalada tomó casi el doble del tiempo en comparación del uso de vara con o sin accesorios en todas sus etapas del aprovechamiento (Tabla 1).

Los grupos de cosecha estuvieron conformados por un cosechador y de uno a tres desgranadores-empaquetadores; generalmente la persona encargada de subir al tronco y cosechar, se incorporaba luego a las actividades de desgranado y empaquetado. Algunos miembros del grupo transportaron los sacos cargándolos al hombro y contratando el servicio de mototaxis hacia el puerto de Villa Jenaro Herrera. La distancia promedio desde el área de aprovechamiento hasta el puerto fue de $1.13 \mathrm{~km}$ (rango: 0.34-9.78 km).

Respecto a la productividad, obtuvimos que un racimo produce en promedio $26.2 \pm 12.48 \mathrm{~kg}$ de frutos de aguaje (rango: 2-56 kg), observándose un total de 1,525.1 kg de frutos aprovechados y 34 sacos durante las evaluaciones (Tabla 2). Cada individuo de aguaje evaluado rindió en promedio 2 sacos de frutos de aguaje (rango: $0.3-4.5$ sacos). El peso promedio de un fruto de aguaje fue de $50.42 \pm 8.90 \mathrm{~g}$ (rango: $35.4-74.22 \mathrm{~g}$ ). No se observó una correlación entre el número de racimos y el peso promedio del fruto por individuo de aguaje $\left(\mathrm{R}^{2}=0.0167\right)$.

\section{DISCUSIÓN}

\section{PANORAMA DEL APROVECHAMIENTO DEL AGUAJE EN JENARO HERRERA}

Nuestro estudio sobre el aprovechamiento de frutos de aguaje en el distrito de Jenaro Herrera describe las características de esta actividad productiva que representa un ingreso adicional al hogar del poblador local; sin embargo, para algunos cosechadores, es la actividad económica más importante. Hemos evidenciado que los cosechadores tienen interés en manejar el aguaje de manera sostenible, precisamente porque reconocen los problemas relacionados a la baja disponibilidad de individuos productivos y las mayores distancias que deben recorrer actualmente para obtener los frutos. Esta es la realidad que viven muchos pobladores que dependen de la extracción de frutos de aguaje en Loreto (Vásquez \& Gentry, 1989), lo cual ha motivado en Jenaro Herrera, la aplicación de prácticas de manejo sostenible a través del uso de técnicas no destructivas de cosecha. Casos similares fueron reportados en otros centros poblados de la Amazonía peruana, como por ejemplo Veinte de Enero, Parinari y Roca Fuerte, donde llevan más de 17 años utilizando el equipo de escalada diseñado por los hermanos Flores (Bejarano \& Piana, 2002; León et al., 2008; Manzi \& Coomes, 2009; Bernal \& Galeano, 2013).

Otra iniciativa de manejo sostenible utilizada por los pobladores de Jenaro Herrera es el cultivo de individuos de aguaje en las áreas de aprovechamiento (Manzi \& Coomes, 2009), pues expone una visión de producción a largo 
plazo, posiblemente, motivada por la escasez de aguajales naturales en las cercanías al pueblo y la creciente demanda del mercado local por los frutos de aguaje. Cabe resaltar la función que cumple la presencia del Centro de Investigaciones Jenaro Herrera del Instituto de Investigaciones de la Amazonía Peruana (CIJHIIAP) donde se viene desarrollando el plan de mejoramiento genético para la domesticación del aguaje (Del Castillo et al., 2010). Este proyecto ha promocionado el establecimiento de plantones de aguaje de alto rendimiento en la zona, cuyos individuos a pesar de no ser $100 \%$ precoces, si son siempre identificados por los pobladores como productores de frutos de alta calidad.

\section{ESTADO DE CONSERVACIÓN DE LAS ÁREAS DE APROVECHAMIENTO}

El paisaje deVilla Jenaro Herrera ha sido moldeado por las actividades productivas impulsadas en el pasado, la ganadería transformó grandes áreas de bosques en pastos, algunos de estos se mantienen como tal hasta la actualidad y otros espacios están en proceso de recuperación. En tal sentido, el poblador local ha demostrado adaptación en el manejo de las áreas de aprovechamiento de aguaje. Por ejemplo, observamos el cuidado y mantenimiento de individuos femeninos en sistemas agroforestales y silvopastoriles en huertas cercanas a la Villa y en predios a lo largo de la carretera Jenaro Herrera - Angamos, así como en fragmentos de aguajales naturales. La capacidad de entendimiento de la escasez del recurso y la voluntad de manejarlo sumado al cultivo del aguaje son medidas recomendadas para lograr el manejo sostenible en la cosecha de frutos de aguaje (Virapongse et al., 2017), y así generar oportunidades para la conservación sin dejar de lado el abastecimiento del mercado.

Las áreas de aprovechamiento utilizadas en el pasado dentro de los aguajales naturales (p.e. Quebrada Fierrillo, Figura 3a) suelen tener una proporción más alta de individuos masculinos sobre individuos femeninos y casi nula regeneración natural, lo cual es un indicador de la fuerte presión que han sufrido estos bosques durante las últimas décadas (Horn et al., 2012). Sin embargo, rescatamos un fragmento de aguajal en particular, en el que el alto número de aguajes juveniles y la similar proporción de individuos masculinos y femeninos (Figura 3c) sugiere que el manejo sostenible puede asegurar un buen estado de conservación de los rodales naturales (Kahn \& De Granville, 1992).

\section{USO DE TÉCNICAS SOSTENIBLES DE APROVECHAMIENTO Y SU IMPLICANCIA EN LA CONSERVACIÓN}

Durante las evaluaciones observamos que los pobladores del distrito de Jenaro Herrera se vienen adaptando a la degradación del recurso, a través del uso de técnicas sostenibles de cosecha. Notamos el interés por el uso del subidor Maquisapa, equipo que fue introducido por el IIAP en la zona de estudio a inicios del 2018. Reportamostambién el uso desus propias técnicas como la vara con o sin accesorios y la pretina; dichas técnicas también han sido observadas en otras comunidades amazónicas pero no se han aplicado consistentemente, resultando en la degradación de los aguajales (Virapongse et al., 2017). El uso de métodos locales de aprovechamiento sostenible tiene como limitante la accesibilidad a los frutos en individuos de aguaje cuando estos alcanzan más de $20 \mathrm{~m}$ de altura, para lo cual es común que recurran a la tala, que aún sigue siendo una alternativa rápida y fácil para algunos cosechadores (Manzi \& Coomes, 2009). En estas circunstancias, es relevante la intervención de entidades gubernamentales y no gubernamentales a través de la transferencia de técnicas no destructivas y seguras para el cosechador (Manzi \& Coomes, 2009). En este sentido, la ejecución de la fase de campo nos 
permitió un acercamiento a los cosechadores locales para continuar difundiendo el uso del subidor Maquisapa como una técnica alternativa de cosecha de individuos productivos con racimos muy elevados. Todos los cosechadores visitados fueron capacitados en el uso adecuado del equipo y recibieron información técnica sobre el manejo sostenible del aguaje por el personal técnico del IIAP.

En el transcurso del estudio tuvimos la oportunidad de conversar con los cuatro principales comerciantes intermediarios en el comercio del aguaje en Villa Jenaro Herrera, quienes manifestaron haber acopiado aproximadamente 1,800 sacos de aguaje en el puerto del pueblo con la finalidad de venderlos en otras ciudades del Bajo Ucayali como Contamana, Juancito, Bretaña, y en pocos casos a Iquitos. De acuerdo a Horn et al. (2018) Jenaro Herrera abasteció a Iquitos con 497 sacos de aguaje en la temporada de cosecha del 2012 (abril - octubre). Trasladando esta cifra al año 2018, Iquitos estaría recepcionando alrededor del $27.6 \%$ de la producción total de frutos de aguaje obtenida en Jenaro Herrera.

Por otro lado, registramos la producción de 34 sacos de aguaje de manera presencial y otros 600 sacos extraídos del fragmento de aguajal manejado por uno los cosechadores más comprometidos con el aprovechamiento sostenible. Esta cifra nos permite afirmar que durante la temporada de cosecha del 2018 al menos el 36\% de la producción total de aguaje en Jenaro Herrera se realizó con técnicas de cosecha no destructivas.

El aprovechamiento de frutos de aguaje es una fuente secundaria de ingresos económicos para la mayoría de los pobladores de Jenaro Herrera; sin embargo, esta actividad productiva cada vez cobra mayor importancia debido a la creciente demanda de los frutos de aguaje a nivel local y regional. Consideramos que es necesario monitorear el uso de técnicas de cosecha no destructivas e incentivar la domesticación del aguaje para permitir la recuperación de las poblaciones en aguajales naturales remanentes. La voluntad de los pobladores del distrito de Jenaro Herrera de manejar el recurso genera nuevas oportunidades para la conservación y manejo sostenible del aguaje, asegurando así el abastecimiento de la demanda.

\section{AGRADECIMIENTOS}

Deseamos agradecer al personal encargado del Centro de Investigaciones Jenaro Herrera por proveer la logística y a Leonardo Ríos Curichimba por su apoyo en el trabajo de campo y por su dedicación a la capacitación de los cosechadores en el uso del equipo Maquisapa. Estamos eternamente agradecidos con los 35 pobladores de Jenaro Herrera, Nuevo Progreso, Nuevo San Juan, Nuevo Aucayacu y Once de Agosto, quienes colaboraron con este estudio compartiendo sus experiencias como cosechadores de aguaje y por su entusiasmo en aprender otras técnicas de cosecha sostenibles.

\section{BIBLIOGRAFÍA CITADA}

Balslev, H.; Grandez, C.; Paniagua, N.; Moller, A.; Hansen, S. 2008. Palmas (Arecaceae) útiles en los alrededores de Iquitos, Amazonía Peruana. Revista Peruana de Biología, 15(1): 121-132.

Bejarano, P.; R. Piana. 2002. Plan de manejo de los aguajales aledaños al caño Parinari. Programa Integral de Desarrollo y Conservación. WWFAIF/DK, Iquitos.

Bernal, R.; Galeano, G. (Eds). 2013. Cosechar sin destruir. Aprovechamiento sostenible de palmas 
colombianas. Facultad de Ciencias, Instituto de Ciencias Naturales. Universidad Nacional de Colombia, Bogotá.

Bodmer, R.E. 1991. Strategies of seed dispersal and seed predation in Amazonian ungulates. Biotropica, 23(3): 255-261.

Brako L.; Zarucchi L.J. 1993. Catalogue of the flowering plants and gymnosperms of Peru, Missouri Botanical Garden. St. Louis, Missouri, USA.

Castaño-Arboleda, N., Cárdenas, D., Rodriguez, E.O., (Eds.), 2007. Ecología, aprovechamiento y manejo sostenible de nueve especies de plantas del departamento del Amazonas, generadoras de productos maderables y no maderables. Instituto Amazónico de Investigaciones Científicas SINCHI, Corporación para el Desarrollo Sostenible del Sur de la Amazonia CORPOAMAZONIA, Ministerio de Ambiente, Vivienda y Desarrollo Territorial, Bogotá, Colombia.

Darnet, S.H.; da Silva, L.H.M.; Rodrigues, A.M.; Lins, R.T. 2011. Nutritional composition, fatty acid and tocopherol contents of buriti (Mauritia flexuosa) and patawa (Oenocarpus bataua) fruit pulp from the Amazon region. Food Science and Technology, 31: 488-491.

Del Castillo, D.; Freitas, L.; García, C.; Sotero, V.; García, D.; Imán, S.; Rojas, R.; Mejía, K.; Gonzáles, A. 2010. Programa de mejoramiento genético del aguaje. Instituto de Investigaciones de la Amazonía Peruana, Innovación y Competitividad para el Agro Peruano.

Delgado, C.; Couturier, G.; Mejía, K. 2007. Mauritia flexuosa (Arecaceae: Calamoideae), an Amazonian palm with cultivation purposes in Peru. Fruits, 62(3): 157-169.

Draper, F. C.; Roucoux, K. H.; Lawson, I. T.; Mitchard, E. T.; Honorio, E. N.; Lähteenoja, O.; Torres Montenegro, L.; Valderrama Sandoval, E.; Zárate, R.; Baker, T. R. 2014. The distribution and amount of carbon in the largest peatland complex in Amazonia. Environmental. Research Letters, 9: 124017. doi:10.1088/17489326/9/12/124017.

Freitas, L. 2012. Impacto del aprovechamiento en la estructura, producción y valor de uso del aguaje en la Amazonía peruana. Recursos Naturales y Ambiente, 67: 35-45.

Galeano, A.; Urrego, L.E.; Sánchez, M.; Peñuela, M.C. 2015. Environmental drivers for regeneration of Mauritia flexuosa L.f. in Colombian Amazonian swamp forest. Aquatic Botany, 123: 47-53.

Henderson, A.; Galeano, G.; Bernal, R. 1995. Field Guide to the Palms of the Americas. Princeton University Press, New Jersey. 352pp.

Hiraoka, M. 1999. Miriti (Mauritia flexuosa) palms and their uses and management among the ribeirinhos of the Amazon estuary. In: Padoch, C.; Ayres, J.M.; Pinedo-Vasquez, M.; Henderson, A. (Eds). Várzea: Diversity, Development, and Conservation of Amazonia's Whitewater Floodplains. New York Botanical Garden, New York, NY, USA. 169-186.

Holm, J.A.; Miller, C.J.; Cropper, W.P. 2008. Population dynamics of the dioecious Amazonian palm Mauritia flexuosa: simulation analysis of sustainable harvesting. Biotropica, 40: 550-558.

Honorio-Coronado, E.N.; Vega-Arenas, J.E.; \& Corrales-Medina, M.N. 2015. Diversidad, estructura y carbono de los bosques aluviales del noreste peruano. Folia Amazónica, 24(1): 55-70.

Horn, C.M.; Gilmore, M.P.; Endress, B.A. 2012. Ecological and socio-economic factors influencing aguaje (Mauritia flexuosa) resource management in two indigenous communities in the Peruvian Amazon. Forest Ecology Management, 267: 93-103.

Horn, C.M.; Vargas, V.; Gilmore, M.P.; Endress, B. 2018. Spatio-temporal patterns of Mauritia flexuosa fruit extraction in the Peruvian 
Amazon: Implications for conservation and sustainability. Applied Geography, 9: 98-108.

Householder, J.E.; Janovec, J.P.; Tobler, M.W.; Page, S.; Lähteenoja, O. 2012. Peatlands of the Madre de Dios river of Peru: distribution, geomorphology, and habitat diversity. Wetlands, 32: 359-368.

Kahn, F.; Mejía K. 1988. Las palmeras nativas de importancia económica en la Amazonía Peruana. Folia Amazónica, 3 (1): 49-59.

Kahn, F.; Moussa F. 1994. Las palmeras del Perú. Inst. Fr. Estad. Andin., IFEA, Lima, Perú.

Kahn, F.; de Granville, J.J. 1992. Palms in forest ecosystems of Amazonia. Springer Verlag, Berlin. 226pp.

Khorsand Rosa, R.; Koptur, S. 2013. New findings on the pollination biology of Mauritia flexuosa (Arecaceae) in Roraima, Brazil: Linking dioecy, wind, and habitat. American Journal of Botany, 100: 13-621.

Kvist, L.P.; Nebel, G. 2001. A review of Peruvian flood plain forests: ecosystems, inhabitants and resource use. Forest Ecology and Management, 150: 3-26.

Lees, A.C.; Peres, C.A. 2008. Conservation value of remnant riparian forest corridors of varying quality for Amazonian birds and mammals. Conservation Biology, 22: 439-449.

León Morales, F.; Del Castillo Torres, D.; Otárola Acevedo, E.; Vega Arenas, J. 2008. Pago por servicios ambientales globales con base en el almacenamiento de carbono de los aguajales de la Reserva Nacional Pacaya Samiria. Instituto de Investigaciones de la Amazonía Peruana.

López Parodi, J.; Freitas, D. 1990. Geographical aspects of forested wetlands in the lower Ucayali, Peruvian Amazonia. Forest Ecology and Management, 33/34: 157-168.

Macedo, R.H. 2002. The avifauna: ecology, biogeography, and behavior. In: Oliveira, P.S., Marquis, R.J. (Eds). The Cerrados of Brazil: Ecology and Natural History of a Neotropical
Savanna. Columbia University Press, New York, USA. 242-265.

Manzi, M.; Coomes, 0. 2009. Managing Amazonian palms for community use: A case of aguaje palm (Mauritia flexuosa) in Peru. Forest Ecology and Management, 257: 510-517.

Pacheco Santos, L. 2005. Nutritional and ecological aspects of buriti or aguaje (Mauritia flexuosa Linnaeus filius): A carotene-rich palm fruit from Latin America. Ecology of Food and Nutrition, 44(5): 345-358.

Padoch C. 1986. Aguaje (Mauritia flexuosa L. f.) in the economy of Iquitos, Peru. Advances in Economic Botany, 6: 214-224.

Padoch C. 1992. Marketing of non-timber forest products in western Amazonia: General observations and research priorities. In: Nepstad, D.; Schwartzman, S. (Eds). Advances in Economic Botany, 9. New York Botanical Gardens, New York.

Phillips, 0. 1993. The potential for harvesting fruits in tropical rainforests: new data from Amazonian Peru. Biodiversity and Conservation, 2: 18-38.

Díaz, J.; M.; Paredes, M.; Martinez, J. 2015. Evaluación participativa de la fenología de las palmeras aguaje (Mauritia flexuosa) y ungurahui (Oenocarpus bataua) en la comunidad nativa Tres Islas, Madre de Dios. Rainforest Alliance. 40pp.

Ríos, M.; Dourojeanni, M.; Tovar, A. 1974. La fauna y su aprovechamiento en Jenaro Herrera (Requena, Perú). Revista Forestal del Perú, 5(1-2): 73-92.

Silva, R.S.; Ribeiro, L.M.; Mercadante-Simões, M.O.; Nunes, Y.R.F.; Lopes, P.S.N. 2014. Seed structure and germination in buriti (Mauritia flexuosa), the Swamp palm. Flora-Morphology, Distribution. Funct. Ecol. Plants, 209: 674-685. Van Lent, J.; Hergoualc'h, K.; Verchot, L.; Oenema, 0.; Willem van Groenigen, J. 2018. Greenhouse gas emissions along a peat swamp forest 
degradation gradient in the Peruvian Amazon: soil moisture and palm roots effects. Mitig. Adapt. Strateg. Glob. Change.

Vásquez, D.; Gentry, A. 1989. Use and misuse of forest-harvested fruits in the Iquitos area. Conservation Biology, 3: 350-361.

Villachica, H.; Urano, de C.J.E.; Hans, M.C.; Díaz, S.C.; Almanza, M. 1996. Frutales y hortalizas promisorias de la Amazonía. Tratado de Cooperación Amazónico. Lima. 367pp.

Virapongse, A.; Endress, B. A.; Gilmore, M. P.; Horn, C.; Romulo, C. 2017. Ecology, livelihoods, and management of the Mauritia flexuosa palm in South America. Global Ecology and Conservation, 10: 70-92.

Recibido: 30 de setiembre de 2018 Aceptado para publicación: 30 de noviembre de 2018 